\title{
Lo mejor del Congreso Virtual ACC/WCC 2020
}

\author{
Dres. Yamel Ache1, Carlos Guamán¹, María Victoria Ramos¹, \\ Víctor Dayan², Jorge Estigarribia²
}

\section{Resumen}

La pandemia generada por el SARS-CoV-2 (Severe Acute Respiratory Syndrome Coronavirus) ha constituido un desafío mundial. En momentos de dificultad como los que se han presentado recientemente, es aún más necesaria la investigación para avanzar en el diagnóstico y tratamiento de las diferentes patologías. Utilizando el amplio desarrollo de los medios de comunicación, este año el Congreso del Colegio Americano de Cardiología (ACC por su sigla en inglés) se celebró del 28 al 30 de marzo en un formato virtual, permitiendo a profesionales de todo el mundo acceder a múltiples actividades científicas con el mismo nivel de excelencia que en las sesiones presenciales.

Realizaremos un breve resumen de alguno de los principales trabajos científicos presentados:

- Tailored Antiplatelet Initiation to Lessen Outcomes Due to Decreased Clopidogrel Response after Percutaneous Coronary Intervention: TAILOR-PCI.

- Rivaroxaban in Peripheral Artery Disease after Revascularization: VOYAGER PAD trial.

- Vericiguat in Patients with Heart Failure and Reduced Ejection Fraction: VICTORIA Study Group.

- The Evolut Low Risk Bicuspid Study.

- PARTNER 3. Two-year clinical and echocardiographic outcomes.

- Ticagrelor with and without Aspirin in Acute Coronary Syndrome After PCI: the TICO Trial.

- Apixaban for the Treatment of Venous Thromboembolism Associated with Cancer: Caravaggio trial.

- Ten-year Outcomes After Drug-eluting Stents Versus Coronary Artery Bypass Grafting for Left Main Coronary Disease: Extended Follow Up of the PRECOMBAT Trial.

- Anticoagulation with or without Clopidogrel after Transcatheter Aortic-Valve Implantation: POPular TAVI.

Palabras clave: $\quad$ TAILOR PCI

VOYAGER PAD

VICTORIA

THE EVOLUT LOW RISK BICUSPID STUDY

PARTNER 3

TICO

CARAVAGGIO

PRECOMBAT

POPULAR-TAVI

\section{The best of the 2020 ACC/WCC Virtual Congress}

\author{
Summary \\ The SARS-CoV-2 pandemic has been a global challenge. In times of difficulty such as those that have recently arisen, re- \\ search is even more necessary to advance in the diagnosis and treatment of different pathologies. Using the extensive de- \\ velopment of the media, this year the American Congress of Cardiology was held from March 28 to 30 in a virtual format, \\ allowing professionals from around the world to access multiple scientific activities with the same level of excellence as \\ in face-to-face sessions. \\ We will make a brief summary of some of the main scientific papers presented: \\ - Tailored Antiplatelet Initiation to Lessen Outcomes Due to Decreased Clopidogrel Response after Percutaneous Coro- \\ nary Intervention: TAILOR-PCI. \\ - Rivaroxaban in Peripheral Artery Disease after Revascularization: VOYAGER PAD trial.
}

1. Editor/a adjunto/a de la Revista Uruguaya de Cardiología.

2. Editor asociado de la Revista Uruguaya de Cardiología.

Correspondencia: Dra. Yamel Ache Tricot. Correo electrónico: yamelache@gmail.com

Los autores declaran no tener conflictos de intereses.

Recibido Mar 28, 2020; aceptado Mar 31, 2020. 
- Vericiguat in Patients with Heart Failure and Reduced Ejection Fraction: VICTORIA Study Group.

- The Evolut Low Risk Bicuspid Study.

- PARTNER 3. Two-year clinical and echocardiographic outcomes.

- Ticagrelor with and without Aspirin in Acute Coronary Syndrome After PCI: the TICO Trial.

- Apixaban for the Treatment of Venous Thromboembolism Associated with Cancer: Caravaggio trial.

- Ten-year Outcomes After Drug-eluting Stents Versus Coronary Artery Bypass Grafting for Left Main Coronary Disease: Extended Follow Up of the PRECOMBAT Trial.

- Anticoagulation with or without Clopidogrel after Transcatheter Aortic-Valve Implantation: POPular TAVI.

$\begin{array}{ll}\text { Key words: } & \text { TAILOR PCI } \\ & \text { VOYAGER PAD } \\ & \text { VICTORIA } \\ & \text { THE EVOLUT LOW RISK BICUSPID STUDY } \\ & \text { PARTNER } 3 \\ & \text { TICO } \\ & \text { CARAVAGGIO } \\ & \text { PRECOMBAT } \\ & \text { POPULAR-TAVI }\end{array}$

\section{O melhor do Congresso Virtual ACC/WCC 2020}

\section{Resumo}

A pandemia de SARS-CoV-2 tem sido um desafio global. Em tempos de dificuldade como os que surgiram recentemente, a pesquisa é ainda mais necessária para avançar no diagnóstico e tratamento de diferentes patologias. Utilizando o amplo desenvolvimento da mídia, este ano o Congresso Americano de Cardiologia foi realizado de 28 ao 30 de março em um formato virtual, permitindo que profissionais de todo o mundo acessassem diversas atividades científicas com o mesmo nível de excelência que nas sessões presenciais.

Faremos um breve resumo de alguns dos principais trabalhos científicos apresentados:

- Tailored Antiplatelet Initiation to Lessen Outcomes Due to Decreased Clopidogrel Response after Percutaneous Coronary Intervention: TAILOR - PCI.

- Rivaroxaban in Peripheral Artery Disease after Revascularization: VOYAGER PAD trial.

- Vericiguat in Patients with Heart Failure and Reduced Ejection Fraction: VICTORIA Study Group.

- The Evolut Low Risk Bicuspid Study.

- PARTNER 3. Two-year clinical and echocardiographic outcomes.

- Ticagrelor with and without Aspirin in Acute Coronary Syndrome After PCI: the TICO Trial.

- Apixaban for the Treatment of Venous Thromboembolism Associated with Cancer: Caravaggio trial.

- Ten-year Outcomes After Drug-eluting Stents Versus Coronary Artery Bypass Grafting for Left Main Coronary Disease: Extended Follow Up of the PRECOMBAT Trial.

- Anticoagulation with or without Clopidogrel after Transcatheter Aortic-Valve Implantation: POPular TAVI.

Palavras chave: TAILOR PCI

VOYAGER PAD

VICTORIA

THE EVOLUT LOW RISK BICUSPID STUDY

PARTNER 3

TICO

CARAVAGGIO

\section{Tailored Antiplatelet Initiation to Lessen Outcomes Due to Decreased Clopidogrel Response after Percutaneous Coronary Intervention: TAILOR - PCI}

El clopidogrel, principal antiagregante utilizado en nuestro medio y el mundo para la doble terapia antiplaquetaria (DAP), tiene metabolización dependiente de la enzima hepática CYP2C19. Hasta un $30 \%$ de la población puede presentar alteraciones genéticas en esta enzima que reducen la capacidad de metabolizar la droga, disminuyendo la disponibi- lidad del fármaco activo y su efecto. La FDA aconseja un tratamiento alternativo en los metabolizadores lentos del CYP2C19 identificados mediante genotipo, pero las sociedades científicas no recomiendan las pruebas genéticas sistemáticas, por no existir estudios prospectivos sobre la implicancia clínica de este fenómeno. 
Presentado por Naveen L. Pereira, Profesor de Medicina de la Clínica Mayo, TAILOR-PCI es un estudio aleatorizado, de superioridad y multicéntrico llevado a cabo en 25 hospitales de Estados Unidos, Canadá, México y Corea. Incluyó 5.302 pacientes que habían recibido un stent coronario por síndrome coronario agudo (SCA) o enfermedad coronaria estable con indicación de DAP por 12 meses, siendo hasta la fecha el mayor estudio genético cardiovascular. El objetivo fue determinar si las pruebas genéticas para identificar portadores de mutaciones de CYP2C19 podían mejorar los resultados de pacientes sometidos a intervención coronaria percutánea (ICP). Los pacientes fueron aleatorizados con proporción 1:1 a terapia convencional o guiada por genotipo. La terapia convencional consistió en clopidogrel $75 \mathrm{mg}$ al día asociado a ácido acetil salicílico, mientras que los portadores de las mutaciones en el grupo guiado por genotipo recibieron ticagrelor $90 \mathrm{mg}$ cada 12 horas. Se excluyeron pacientes menores de 18 años, con estenosis pendientes de ser revascularizadas en los 12 meses del procedimiento índice y con genotipo de CYP2C19 conocido previo al inicio del estudio.

El punto final primario fue un compuesto de muerte cardiovascular, infarto de miocardio, accidente cerebrovascular, trombosis del stent probable o definitiva e isquemia recurrente durante el año posterior a la ICP. El punto final secundario evaluó seguridad mediante sangrado mayor o menor definido por los criterios TIMI. Los puntos finales fueron evaluados al alta hospitalaria, al mes, y a los 6 y 12 meses tras la ICP, mediante entrevista telefónica o revisión de registros médicos.

Del total de pacientes, 2.641 fueron randomizados a terapia guiada por genotipo y 2.635 a tratamiento convencional. Del primer grupo, 903 eran portadores de las mutaciones genéticas y fueron comparados al final del seguimiento con 946 portadores de mutaciones asignados al azar a recibir tratamiento convencional. La edad media fue 62 años, siendo $27 \%$ diabéticos. Un $84 \%$ tenía indicado DAP por SCA. El punto final primario compuesto se produjo en $4 \%(\mathrm{n}=35)$ de los portadores del grupo guiado por genotipo y $5,9 \%(\mathrm{n}=54)$ de los portadores que recibieron terapia convencional. Esto significa que no se logró demostrar diferencia significativa (HR: 0,66; IC: 0,43-1,02; $\mathrm{p}=0,056$ ). Tampoco hubo diferencias en hemorragia mayor o menor TIMI, que se presentó en $1,9 \%(\mathrm{n}=16)$ de los portadores con tratamiento guiado y en $1,6 \%(\mathrm{n}=14)$ de los portadores con tratamiento convencional al año. No obstante, un análisis post hoc (para el cual el estudio no fue diseñado), evidenció que hubo diferencias en los primeros 3 meses de DAP con reducción de 79\% del punto final primario en los portadores asignados a terapia guiada por genotipo (HR: 0,21; $\mathrm{p}=0,001$ ). Asimismo, en un análisis de sensibilidad encontraron que los portadores con terapia guiada por genotipo presentaron una reducción de $40 \%$ del riesgo de eventos primarios respecto a aquellos con tratamiento convencional (HR: 0,60; IC: 0,41-0,89; $\mathrm{p}=0,011$ ). Sin duda, lejos de poner un punto final a la polémica de la farmacogenética, estos resultados motivan a la comunidad científica a diseñar nuevos trabajos al respecto.

\section{Bibliografía}

Pereira, N. Mayo Clinic. Clinical Implementation Of Clopidogrel Pharmacogenetics: The Tailor PCI Trial [Internet]. 2020. [citado 28 marzo 2020] Disponible en: https://www.webcastregister.live/accanywhere20livestream/livestreamacc/0361c4d0763f4e779abe596a0afe1d761d

\section{Rivaroxaban in Peripheral Artery Disease after Revascularization (VOYAGER PAD trial)}

Aproximadamente 200 millones de personas a nivel mundial presentan enfermedad arterial periférica (EAP) de miembros inferiores (MMII)(1). Los pacientes con EAP sintomática o asintomática, tienen un aumento de su morbilidad y mortalidad. Después de 5 años del diagnóstico, $20 \%$ de los pacientes con claudicación intermitente tienen un infarto de miocardio (IAM) o accidente cerebrovascular (ACV), con una mortalidad del 10\%-15\%(2). En ensayos clínicos de pacientes con EAP sintomática se ha encontrado una prevalencia de enfermedad coronaria sintomática del $30 \%$ y de historia de IAM de 10\%-20\%, a pe- sar de presentar varios factores de riesgo cardiovasculares $^{(2)}$.

Aquellos pacientes en quienes se realiza revascularización periférica presentan alto riesgo de complicaciones vasculares, particularmente isquemia aguda de la extremidad, la cual se ha asociado con hospitalización prolongada y alta incidencia de pérdida de la extremidad, invalidez y muerte. Actualmente ninguna terapia antitrombótica ha demostrado disminuir el riesgo en este escenario. El estudio COMPASS ${ }^{(4)}$ encontró que rivaroxabán a una dosis de 2,5 mg dos veces al día más ácido acetil 
salicílico, redujo el riesgo de isquemia, incluyendo el riesgo de eventos adversos mayores que afectaban las extremidades en una población con EAP crónica. En base a estas observaciones el Dr. Marc Bonaca y colaboradores diseñaron la hipótesis de que rivaroxabán 2,5 mg dos veces al día más ácido acetilsalicílico $100 \mathrm{mg}$ al día, podrían reducir el riesgo compuesto de isquemia aguda de la extremidad, amputaciones mayores por causas vasculares, IAM, ACV isquémico, o muerte por causa cardiovascular en pacientes con EAP sintomática sometidos a revascularización de los MMII, en comparación con el tratamiento aislado con ácido acetilsalicílico.

VOYAGER PAD ${ }^{(1)}$ es un estudio randomizado, paralelo y estratificado. Sus criterios de inclusión fueron edad $\geq 50$ años, EAP de MMII (documentada por síntomas de isquemia, imagen e índice anormal tobillo-brazo), siendo elegibles luego de un procedimiento exitoso de revascularización dentro de los 10 días previos. Sus criterios de exclusión fueron revascularización por enfermedad asintomática, revascularización reciente ( $<10$ días), isquemia aguda de la extremidad reciente ( $<2$ semanas), o síndrome coronario agudo reciente ( $<30$ días), pérdida importante de tejido actual, necesidad de antiagregantes plaquetarios o anticoagulantes que no sean ácido acetilsalicílico y/o clopidogrel, necesidad de terapia antiplaquetaria doble a largo plazo (clopidogrel podía ser administrado hasta 6 meses luego de la revascularización a criterio del investigador), y alto riesgo de sangrado. Bayer participó en el diseño del estudio, su supervisión, selección, y en la redacción del manuscrito. El Centro de Prevención de Colorado y el Comité Ejecutivo, que incluía empleados de los patrocinadores, fueron responsables del diseño y supervisión del estudio, interpretación de datos y redacción del manuscrito.

El estudio randomizó 6.564 pacientes desde agosto 2015 hasta enero 2018 en 542 centros de 34 países, el seguimiento tuvo una mediana de 28 meses. De estos pacientes, $1.080(33,2 \%)$ en el grupo de rivaroxabán y $1.011(31,1 \%)$ en el grupo placebo, discontinuaron el tratamiento prematuramente. Se incluyeron en el análisis del objetivo primario por intención de tratar 3.286 pacientes en el grupo rivaroxabán y 3.278 pacientes en el grupo placebo, y en el análisis del objetivo de seguridad 3.256 pacientes en el grupo rivaroxabán y 3.248 pacientes en el grupo placebo. En ambos grupos la mediana de la edad fue 67 años, $26 \%$ sexo femenino, $40 \%$ diabéticos, $35 \%$ fumadores actuales, $32 \%$ enfermedad coronaria, $11 \%$ IAM previo, $51 \%$ recibió clopidogrel y $80 \%$ recibió estatinas. En el grupo de rivaroxabán la indicación de revascularización fue claudicación en $77 \%$ e isquemia crítica en $23 \%$ de los casos. El tipo de revascularización fue quirúrgica en $35 \%$ y endovascular o híbrida en $66 \%$ de los casos.

El objetivo primario (compuesto de isquemia aguda de la extremidad, amputación mayor por causa vascular, IAM, ACV isquémico, o muerte por causa cardiovascular) ocurrió en 508 pacientes en el grupo rivaroxabán y en 584 pacientes en el grupo placebo, con una incidencia estimada a los 3 años de $17,3 \%$ y $19,9 \%$, respectivamente (HR: 0,85, IC: 0,76-0,96; $\mathrm{p}=0,0085$ ); las curvas de Kaplan-Meier se separaron a los 3 meses de iniciado el tratamiento con un NNT de 39 para el grupo de rivaroxabán a los 3 años. Al analizar los componentes del objetivo primario sólo se encontró diferencia estadísticamente significativa en la isquemia aguda de la extremidad, 5,24\% en el grupo de rivaroxabán y 7,74\% en el grupo placebo (HR: 0,67, IC: 0,55-0,82).

Durante el seguimiento el objetivo primario de seguridad, sangrado mayor TIMI, ocurrió en 62 pacientes $(2,65 \%)$ en el grupo rivaroxabán y en 44 pacientes $(1,87 \%)$ en el grupo placebo (HR: 1,43; IC: $0,97-2,10 ; p=0,07)$. Ocurrió sangrado fatal en 6 pacientes de cada grupo. El objetivo compuesto de hemorragia intracraneal o sangrado fatal ocurrió en 17 pacientes en el grupo rivaroxabán y en 19 pacientes en el grupo placebo (HR: 0,91; IC: 0,47-1,76). El objetivo secundario de seguridad de sangrado mayor ISTH ocurrió en 140 pacientes $(5,94 \%)$ en el grupo rivaroxabán y en 100 pacientes $(4,06 \%)$ en el grupo placebo (HR: 1,42; IC: 1,10-1,84; p=0,007). El sangrado BARC de grado 3b o mayor ocurrió en 93 pacientes del grupo rivaroxabán y en 73 pacientes del grupo placebo (HR: 1,29; IC: 0,95-1,76; $\mathrm{p}=0,10$ ).

Los investigadores estimaron que por cada 10.000 pacientes tratados durante 1 año con rivaroxabán 2,5 mg dos veces al día y ácido acetilsalicílico 100 mg día, podrían prevenirse 181 eventos del objetivo primario a costo de 29 eventos del objetivo primario de seguridad.

La principal limitación del estudio fue el porcentaje de pacientes que discontinuaron el tratamiento prematuramente (aproximadamente 14\% por año en el grupo de rivaroxabán), mayor que lo anticipado por los investigadores.

Finalmente se puede interpretar que la combinación de rivaroxabán a dosis baja y ácido acetilsalicílico se asoció con una reducción del riesgo absoluto de $2,6 \%$ en muerte por causa cardiovascular, isquemia aguda de la extremidad, amputación mayor, IAM o ACV isquémico en comparación con el uso aislado de ácido acetilsalicílico, luego de 3 años. A pesar de que la combinación se asoció a mayor sangrado, la tasa de sangrado intracraneal fue similar en ambos grupos, hecho destacado por el Dr. Marc Bonaca durante su presentación ${ }^{(1)}$. 
El Dr. Allan Brett comentó que la inspección de las curvas de eventos en el artículo sugiere que la mayor parte del beneficio acumulado ocurre durante el primer año de seguimiento, por lo tanto, la duración óptima del tratamiento sigue sin estar clara. Después de uno o dos años, los riesgos de sangrado podrían comenzar a superar los beneficios ${ }^{(5)}$.

\section{Bibliografía}

1. Bonaca M, Bauersachs R, Anand S, Debus ES, Nehler M, Patel M, et al. Rivaroxaban in Peripheral Artery Disease after Revascularization. N Engl J Med 2020 Mar 28; [e-pub]. doi: 10.1056/NEJMoa 2000052

2. Weitz JI, Byrne J, Clagett GP, Farkouh ME, Porter JM, Sackett DL, et al. Diagnosis and treatment of chronic arterial insufficiency of the lower ex- tremities: a critical review. Circulation. 1996;94: 3026-49. doi: 10.1161/01.cir.94.11.3026

3. Hiatt WR, Fowkes FG, Heizer G, Berger JS, Baumgartner I, Held P, et al. Ticagrelor versus Clopidogrel in Symptomatic Peripheral Artery Disease. N Engl J Med. 2017 Jan 5;376(1):32-40. doi: 10.1056/NEJMoa1611688

4. Eikelboom JW, Connolly SJ, Bosch J, Dagenais GR, Hart RG, Shestakovska O, et al. Rivaroxaban with or without Aspirin in Stable Cardiovascular Disease. N Engl J Med. 2017 Oct 5;377(14):1319-30. doi: 10.1056/NEJMoa1709118

5. Brett A [Internet]. Marzo 28, 2020. Anticoagulation After Lower-Limb Revascularization?. [Consulta 28, marzo, 2020]. Disponible en: https://www. jwatch.org/na51205/2020/03/28/anticoagulation-after-lower-limb-revascularization

\section{Vericiguat in Patients with Heart Failure and Reduced Ejection Fraction: VICTORIA Study Group}

Se ha demostrado que los pacientes con insuficiencia cardíaca (IC) presentan afectación de la vía óxido nítrico $(\mathrm{ON})$ - guanilato ciclasa soluble (GCs) guanosín monofosfato cíclico (GMPc), con el consecuente deterioro en sus funciones vasodilatadoras y antifibróticas, entre otras. El descubrimiento de moléculas capaces de estimular la GCs, como el vericiguat ha despertado interés en evaluar su eficacia y seguridad en el tratamiento de la IC con fracción de eyección reducida.

VICTORIA $^{(1)}$ es un ensayo fase III, multicéntrico, randomizado, doble ciego y controlado por placebo. Contó con financiación del Centro Canadiense VIGOUR, Merck y Bayer. Desde septiembre de 2016 hasta diciembre 2018 se incluyeron pacientes mayores de 18 años con IC clase funcional II-IV de la New York Heart Association (NYHA), fracción de eyección del ventrículo izquierdo (FEVI) $\leq 45 \%$ y niveles elevados de péptidos natriuréticos (péptido natriurético tipo-B $[\mathrm{BNP}] \geq 300 \mathrm{pg} / \mathrm{ml}$ en ritmo sinusal $\mathrm{y} \geq 500 \mathrm{pg} / \mathrm{ml}$ en fibrilación auricular o fracción amino-terminal de propéptido natriurético tipo $\mathrm{B}[\mathrm{NT}$-proBNP $] \geq 1.000 \mathrm{pg} / \mathrm{ml}$ $\mathrm{y} \geq 1.600 \mathrm{pg} / \mathrm{ml}$ en ritmo sinusal y fibrilación auricular respectivamente). También debían presentar empeoramiento de la IC, y se clasificaron en tres cohortes de acuerdo al momento del deterioro: los hospitalizados dentro de los tres meses anteriores a la randomización, los hospitalizados de tres a seis meses previos, y aquellos que reciben terapia diurética intravenosa, sin hospitalización dentro de los tres meses anteriores. Se excluyeron aquellos con presión arterial sistólica $<100$ $\mathrm{mmHg}$, uso actual o anticipado de nitratos, estimulan- tes de la GCs o inhibidores de la fosfodiesterasa tipo 5, inestabilidad clínica al momento de la randomización (inotrópicos intravenosos o dispositivo de asistencia ventricular izquierda), enfermedad valvular primaria que requiriera tratamiento intervencionista, miocardiopatía hipertrófica, miocarditis, endocarditis activa, síndrome coronario agudo o intervención coronaria en los 60 días previos a la randomización, y enfermedad pulmonar severa, entre otras.

Se randomizaron 5.050 pacientes $1: 1$ a vericiguat $2,5 \mathrm{mg}$ versus placebo $2,5 \mathrm{mg}$. Las dosis se incrementaron hasta un máximo de $10 \mathrm{mg}$ una vez al día. El seguimiento se realizó a las 2 y 4 semanas, y posteriormente cada 4 meses hasta la finalización del estudio. Las características basales de la población fueron comparables en ambos grupos: edad media 67 años, $24 \%$ sexo femenino, $40 \%$ se presentaba en clase funcional III NYHA y FEVI media $29 \%$. En cuanto al uso concomitante de terapia basada en las guías de práctica clínica, $60 \%$ recibía triple terapia (betabloqueantes, antagonistas de mineralocorticoides e inhibidores de la enzima conversora de angiotensina, bloqueante del receptor de angiotensina o sacubitrilo-valsartán). Un $32 \%$ de los pacientes presentaban dispositivos (desfibrilador y/o resincronizador). La adherencia a la droga de estudio (vericiguat o placebo) fue mayor de $80 \%$.

Los resultados fueron presentados por el Dr. Paul Armstrong ${ }^{(2)}$ y publicados simultáneamente en The New England Journal of Cardiology ${ }^{(1)}$. Durante el seguimiento 610 pacientes del grupo vericiguat y 565 del grupo placebo abandonaron el régi- 
men de tratamiento. El objetivo primario (compuesto de muerte por causa cardiovascular o primer hospitalización por IC) fue 35,5\% en el grupo vericiguat versus $38,5 \%$ en el placebo (HR: 0,90; IC: $0,82-0,98 ; \mathrm{p}=0,02)$ a expensas de un descenso de la hospitalización por IC (HR: 0,90; IC: 0,81-1,00; $\mathrm{p}=$ $0,04)$, siendo la reducción absoluta de eventos 4,2/100 pacientes/año. En cuanto a los objetivos secundarios, no se observaron diferencias estadísticamente significativas en muerte cardiovascular, mortalidad por todas las causas o eventos adversos (hipotensión sintomática o síncope).

Como conclusión los autores destacan que Vericiguat aborda un nuevo objetivo terapéutico, con buen perfil de seguridad y tolerancia, sin necesidad de controlar la función renal y los electrolitos.

“... Vericiguat proporciona una novedosa adición al tratamiento habitual”(3) expresó Armstrong. $\mathrm{Y}$ agregó "creo que es un resultado gratificante en pacientes con insuficiencia cardíaca de alto riesgo que no solo les abre una nueva vía, sino también un

\section{The Evolut Low Risk Bicuspid Study}

En el día de ayer se presentó en el ACC/WCC Virtual 2020, un ensayo clínico financiado por la empresa Medtronic, de un solo brazo, sobre implante percutáneo de válvula aórtica (TAVI) en pacientes con estenosis aórtica severa sobre válvula bicúspide (VAB), sintomáticos o asintomáticos. Dichos pacientes han sido sistemáticamente excluidos en ensayos clínicos anteriores debido a múltiples razones como ser: asimetría en la calcificación, forma elíptica del anillo aórtico, riesgo potencial de expansión incompleta de la prótesis, y problemas técnicos.

El objetivo primario del ensayo fue evaluar la seguridad y eficacia del TAVI en pacientes con VAB y bajo riesgo. La valoración de la morfología valvular se realizó previamente mediante TAC multicorte.

Se excluyeron:

- pacientes menores de 60 años;

- diámetro de la aorta ascendente $>4,5 \mathrm{~cm}$;

- enfermedad coronaria con SYNTAX score $>22$;

- aortopatía con indicación de cirugía;

- calcificación severa del tracto de salida del ventrículo izquierdo.

Como objetivo primario de seguridad se evaluó mortalidad o ataque cerebrovascular (ACV) de cualquier causa a 30 días. Del total de pacientes incluidos inicialmente se excluyeron 60 , resultando un número final de 150 pacientes. La media de edad fue 70 años y un $72,7 \%$ de los pacientes se encontraba en clase funcional NYHA I/II. camino para el descubrimiento futuro de la enfermedad cardíaca"(3).

\section{Bibliografía}

1. Armstrong P, Pieske B, Anstrom K, Ezekowitz J, Hernandez A, et al. Vericiguat in Patients with Heart Failure and Reduced Ejection Fraction. [Internet, consulta 28 de marzo]. Disponible en: nejm. org/doi/pdf/10.1056/NEJMoa1915928

2. The VICTORIA (Vericiguat Global Study in Subjects with Heart Failure with Reduced Ejection Fraction) Trial. [Internet, Consulta 28 de marzo]. Disponible en: acc.mediasite.com/mediasite/play/0361c4d0763f4e779abe596a0afe1d761?autoStart $=$ true

3. Vericiguat Global Study in Subjects with Heart Failure with Reduced Ejection Fraction - VICTORIA. [Internet, Consulta 28 de marzo]. Disponible en: acc.org/latest-in-cardiology/clinical-trials/2020/03/26/19/43/victoria

A 30 días de seguimiento los resultados fueron los siguientes:

- muerte o ACV invalidante: $1,3 \%$;

- ACV no invalidante: $3,3 \%$;

- requerimiento de marcapaso definitivo: $14,7 \%$;

- fuga paravalvular moderada o severa: $0 \%$;

- fuga paravalvular leve: $40,4 \%$.

Los autores concluyen que los resultados a 30 días reflejan la seguridad del procedimiento aunque se debe esperar el seguimiento a 10 años para tener la evidencia suficiente que permita modificar la práctica clínica.

\section{Análisis}

El presente estudio representa un gran avance en el tratamiento de la patología valvular aórtica. Los pacientes con VAB representan una población frecuente (tan frecuente como la tricúspide en los pacientes de 65-70 años). El diagnóstico de la morfología valvular depende principalmente de la valoración imagenológica dentro de la cual se postula como gold standard la tomografía multicorte. Sin embargo, aun con esta técnica, existe un porcentaje importante de falsos diagnósticos al momento de la confirmación quirúrgica.

Este ensayo tiene como fortaleza la selección meticulosa de pacientes a incluir en el estudio con el objetivo de asegurar los mejores resultados. Sin em- 
bargo, esta misma característica es su principal defecto. La selección tan detallada de la población incluida, hace que su generalización y por lo tanto validez externa sea muy limitada. Los resultados quirúrgicos en la sustitución valvular aórtica de pacientes con VAB y jóvenes (menores de 60 años) es muy buena, como evidencia el ensayo clínico RECOVERY publicado recientemente en The New England Journal of Medicine.

En el ensayo presentado en el día de ayer, en un seguimiento de tan solo 30 días, 4,6\% de los pacientes presentaron $\mathrm{ACV}, 14,7 \%$ requirieron marcapaso permanente y $40,4 \%$ quedaron con fuga paravalvular leve siendo pacientes jóvenes. Estos tres elementos ponen precaución sobre el uso actual de esta técnica.

\section{Bibliografía}

1. Kang DH, Park SJ, Lee SA, Lee S, Kim DH, Kim HK, et al. Early Surgery or Conservative Care for Asymptomatic Aortic Stenosis. N Engl J Med. 2020 Jan 9;382(2):111-9. doi: 10.1056/NEJMoa1912846

\section{PARTNER 3. Two-year clinical and echocardiographic outcomes}

En el día de ayer se presentó uno de los ensayos clínicos mas esperados del año en lo referente a patología estructural cardíaca. Se trata del estudio PARTNER 3 y de los resultados de su seguimiento a dos años. El mismo es un ensayo clínico randomizado de 1.000 pacientes con estenosis aórtica severa sintomática de bajo riesgo quirúrgico. En el mismo, los pacientes fueron randomizados a sustitución valvular aórtica quirúrgica (SVAQ) o percutánea (TAVI). El objetivo primario fue la incidencia compuesta de muerte, ataque cerebrovascular (ACV) o rehospitalización al año. Los resultados a un año fueron favorables para TAVI en el objetivo primario y en cada uno de los componentes del mismo. A continuación describiremos los principales hallazgos reportados en cuanto a los resultados a 2 años.

Se analizó un total de 950 pacientes según protocolo (no por intención de tratar).

Seguimiento a 2 años

- TAVI: $99,0 \%$.

- SVAQ: $93,8 \%$.

Objetivo primario (muerte, ACV o rehospitalización)

- TAVI: $11,5 \%$.

- SVAQ: $17,4 \%$. $\mathrm{HR}=0,63 ;$ IC: $0,45-0,68(\mathrm{p}=0,007)$.

Muerte

- TAVI: $2,4 \%$.

- SVAQ: $3,2 \%$. $\mathrm{HR}=0,75 ; \mathrm{IC}: 0,35-1,63(\mathrm{p}=0,47)$.

ACV

- TAVI: $2,4 \%$.

- SVA: $3,6 \%$. $\mathrm{HR}=0,66$; IC: $0,31-1,40(\mathrm{p}=0,28)$.

Muerte o ACV

- TAVI: $3,0 \%$.

- SVAQ: $3,8 \%$.
$\mathrm{HR}=0,77$;C: $0,39-1,55(\mathrm{p}=0,47)$.

Rehospitalización

- TAVI: $8,5 \%$.

- SVAQ: $12,5 \%$.

$\mathrm{HR}=0,67 ; \mathrm{IC}: 0,45-1,00(\mathrm{p}=0,046)$.

La incidencia de fibrilación auricular fue mayor en pacientes con SVAQ $(41,8 \%$ vs $7,9 \%, \mathrm{p}<0,001)$ y la tasa de trombosis valvular fue mayor en pacientes con TAVI $(2,6 \%$ vs $0,7 \%, \mathrm{p}=0,02)$.

La incidencia de fuga paravalvular leve fue mayor en pacientes con TAVI ( $26 \%$ vs $2,3 \%$, p < 0,001 ).

Como conclusión los autores mencionan que TAVI a 2 años sigue siendo superior en cuanto al objetivo primario combinado, sin embargo deja de ser superior (pasa a ser similar) en cuanto a muerte y ACV. Durante el periodo de 1 a 2 años hubo un aumento en la incidencia de ACV y muerte en el grupo TAVI que disminuyen la diferencia con SVAQ y por lo tanto se pierde la diferencia significativa que se veía al año de seguimiento.

\section{Análisis}

Recordemos que el PARTNER 3 es un ensayo clínico en pacientes de bajo riesgo, no de población de pacientes jóvenes. Los resultados reportados al día de hoy se aplican a la evolución a dos años. En otras palabras, si tenemos un paciente con una expectativa de vida de 2 años con estenosis aórtica severa sintomática, es preferible colocar TAVI dado que tendrá menor tasa de rehospitalizacion.

El ensayo clínico tiene un poder estadístico suficiente para definir el beneficio o no del resultado primario compuesto que es: muerte, ACV o rehospitalización al año. De los tres componentes del resultado compuesto, la rehospitalización representa la variable mas controversial y en la cual su atribución 
no es ciega. ¿Qué quiere decir eso? Un paciente del ensayo que se presentó a emergencia y consultó por falta de aire podría ser manejado de forma distinta por el profesional que lo atiende, si fue operado o si se le colocó TAVI. Por lo tanto, la atribución a rehospitalización (que la realiza el médico que atiende al paciente) no es ciega, ya que el mismo conoce de antemano a qué grupo fue randomizado. Cuando se incluye en el resultado primario combinado una variable de ese tipo, aumenta tres veces la probabilidad que el objetivo combinado sea significativo en la rama experimental. Por lo tanto es clave valorar los resultados individuales de las variables componentes de la variable compuesta. En este caso, a diferencia de lo ocurrido en el seguimiento al año, no hay diferencia en las variables duras entre los grupos (muerte, ACV). La única excepción es la rehospitalización, la cual presenta un intervalo de confianza que incluye el 1 , pero sin embargo encontramos que el valor p es igual a 0,045. Destacamos que la incidencia de fuga paravalvular es baja en comparación con otros estudios, pero sigue siendo preocupante (26\% de fuga paravalvular leve) si consideramos aplicar esta técnica a pacientes jóvenes (no fue la población objetivo del estudio). La tasa de incidencia de fibrilación auricular en la rama quirúrgica es excesivamente alta, interesaría saber si se incluyen todos los pacientes que presentaron fibrilación auricular transitoria y permanente, o solamente a quienes quedaron con fibrilación auricular permanente.

Por lo anterior deberíamos concluir de los resultados a dos años del PARTNER 3 que el uso de TAVI en pacientes de bajo riesgo tiene su lugar, siempre y cuando estemos considerando resultados a 2 años. El comportamiento en muerte y ACV entre uno y dos años de seguimiento en el grupo TAVI hace que debamos ser cautos a la hora de aplicar estos resultados en una población con mayor expectativa de vida. El beneficio de TAVI con respecto a la SVAQ a los dos años de seguimiento, es exclusivamente una menor rehospitalización.

\section{Bibliografía}

1. Mack MJ, Leon MB, Thourani VH, Makkar R, Kodali SK, Russo M, et al; PARTNER 3 Investigators. Transcatheter Aortic-Valve Replacement with a Balloon-Expandable Valve in Low-Risk Patients. N Engl J Med. 2019;380(18):1695-705. doi: 10.1056/NEJMoa1814052

2. Freemantle N, Calvert M. Composite and surrogate outcomes in randomised controlled trials. BMJ 2007; 334: 756 .

\section{Ticagrelor with and without Aspirin in Acute Coronary Syndrome After $\mathrm{PCl}$ : the TICO Trial}

Uno de los aspectos más estudiados en los últimos años es la estrategia óptima de antiagregación en los pacientes con síndrome coronario agudo (SCA) e intervención coronaria percutánea (ICP), de forma que permita disminuir la trombosis del stent, con la menor incidencia posible de sangrados. Múltiples ensayos clínicos han intentado definir la mejor combinación de fármacos y el tiempo mínimo que deben ser administrados, persistiendo dudas al respecto.

El ensayo TICO ${ }^{(1)}$ se diseñó sobre la hipótesis de que en pacientes que presentaron SCA y recibieron stents de última generación, el uso de ticagrelor en monoterapia luego de 3 meses de doble antiagregación plaquetaria (DAPT) podría ser superior a la estrategia actual de 12 meses de DAPT.

Se trata de un estudio prospectivo, randomizado y multicéntrico (38 centros de Corea) que incluyó desde agosto de 2015 a octubre de 2018 , pacientes $\geq 19$ años con SCA que recibieron ICP con stent liberador de sirolimus y polímero bio-reabsorbible. Se excluyeron: a) aquellos > 80 años, b) que presentaran riesgo incrementado de sangrado, c) necesidad de anticoagula- ción oral, d) disfunción hepática moderada a severa o e) síntomas dependientes de bradicardia.

El objetivo primario fue definido como la ausencia de eventos clínicos adversos netos (NACE) a 12 meses: un combinado de sangrado mayor TIMI y eventos adversos mayores cardíacos y cerebrovasculares (MACCE) que incluía mortalidad por todas las causas, infarto agudo de miocardio, trombosis del stent, ataque cerebrovascular.

Se randomizaron 3.056 pacientes en relación 1:1 a 3 meses de DAPT (ácido acetilsalicílico + ticagrelor) seguido de monoterapia con ticagrelor hasta completar 12 meses desde el SCA versus el tratamiento convencional en base a DAPT por 12 meses. Las características basales de la población fueron comparables: edad media $61 \pm 11$ años, predominio de sexo masculino (80\%), 50\% de portadores de hipertensión arterial y $27 \%$ de diabetes mellitus. En cuanto a la presentación clínica, aproximadamente un tercio se presentó con angina inestable, un tercio con SCA sin elevación del ST y otro tercio con elevación del ST. Un 55\% presentaba lesión de dos o tres vasos. 
Los resultados fueron presentados por el Dr. Yangsoo Yang. El objetivo primario se alcanzó en $3,9 \%$ en el grupo de monoterapia versus $5,9 \%$ en el grupo convencional (HR: 0,66; IC: 0,48-0,92; $p=0,01$ ) a expensas de una reducción significativa en los sangrados mayores: $1,7 \%$ en el grupo de monoterapia versus $3,0 \%$ en el grupo convencional (HR: 0,56; IC: 0,34-0,91; $\mathrm{p}=0,02)$. Cuando se realiza el análisis a partir de los tres meses de la randomización, las diferencias son aún más marcadas. No se observaron diferencias en cuanto a MACCE $(p=0,09)$. En el análisis por subgrupos la estrategia de monoterapia fue superior al tratamiento convencional, excepto en aquellos pacientes con enfermedad multivaso.

Dentro de las limitaciones del estudio se destaca que no fue controlado con placebo, se excluyeron pacientes con alto riesgo de sangrado no pudiendo extrapolar los resultados a este grupo, y que los eventos del objetivo primario (MACCE y sangrado mayor) fueron menores a lo anticipado.
Sin embargo, cabe destacar que este es el primer estudio randomizado en comparar el tratamiento convencional versus monoterapia con ticagrelor.

"Nuestros hallazgos sugieren que para los pacientes con SCA que reciben tratamiento con stents, la monoterapia con ticagrelor podría ser una estrategia óptima para reducir el riesgo de sangrado sin aumentar el riesgo de eventos adversos isquémicos", expresó Yang.

\section{Bibliografía}

1. Ticagrelor with and without Aspirin in Acute Coronary Syndrome After PCI: Randomized Evaluation of Ticagrelor Monotherapy after 3-month Dual-antiplatelet Therapy in Acute Coronary Syndrome. The TICO Trial. [Internet, Consulta 30 de marzo]. Disponible en: acc.mediasite.com/mediasite/play/b6c8a $7 \mathrm{c} 6 \mathrm{fdd} 664 \mathrm{~b} 6597 \mathrm{c} 8 \mathrm{e} 19255 \mathrm{c} 5 \mathrm{c} 9 \mathrm{ce} 1 \mathrm{~d}$ ?autoStart=true

\section{Apixaban for the Treatment of Venous Thromboembolism Associated with Cancer (Caravaggio trial)}

Los pacientes con cáncer tienen cuatro a siete veces mayor probabilidad de desarrollar tromboembolia venosa (TEV), siendo la trombosis asociada al cáncer la segunda causa de muerte en estos pacientes, luego de la progresión de su enfermedad ${ }^{(1)}$. El tratamiento de la TEV en este escenario es complejo. Recientemente fueron incluidos el rivaroxabán y el edoxabán en las recomendaciones para el tratamiento inicial, siempre que no exista alto riesgo de sangrado gastrointestinal o urinario ${ }^{(2)}$. El Dr. Giancarlo Agnelli y colaboradores, evaluaron la no inferioridad del uso de apixabán frente a heparina de bajo peso molecular (dalteparina subcutánea [SC]), para la prevención del TEV recurrente en pacientes con cáncer sin aumentar el riesgo de hemorragia mayor.

Para probar esta hipótesis diseñaron el estudio Caravaggio $^{(3)}$, multicéntrico, randomizado, controlado, iniciado por el investigador, abierto, de no inferioridad, con adjudicación ciega de los resultados, y con fondos sin restricciones de la alianza Bristol-Myers Squibb-Pfizer (no tuvo rol en la conducción del estudio ni en el diseño del manuscrito). Efectuaron una randomización 1:1 a recibir por 6 meses apixabán (10mg dos veces al día los primeros 7 días, seguido de $5 \mathrm{mg}$ dos veces al día) o dalteparina (200 $\mathrm{UI} / \mathrm{kg}$ SC una vez al día el primer mes, seguido de $150 \mathrm{UI} / \mathrm{kg}$ una vez al día). Las drogas fueron discontinuadas temporalmente en caso de conteo de plaquetas $<50.000 / \mathrm{mcl}$ o de una condición asociada a au- mento del riesgo de sangrado. Los criterios de inclusión fueron: a) TEV sintomática o incidental proximal al miembro inferior, b) embolia pulmonar sintomática, o c) embolia pulmonar incidental en un segmento o más, proximal a la arteria pulmonar, en pacientes con cualquier tipo de cáncer excepto carcinoma de la piel (de células basales o de células escamosas), tumor cerebral primario o metástasis cerebral conocida, y leucemia aguda. Los criterios mayores de exclusión fueron: a) edad $<18$ años, b) estadio del grupo de oncología cooperativa del este III o IV, c) expectativa de vida $<6$ meses, d) dosis terapéuticas de heparina de bajo peso molecular, e) heparina no fraccionada o antagonista de la vitamina $\mathrm{K}>72$ horas antes de la randomización, f) otra indicación de anticoagulación, g) uso concomitante de antiplaquetarios (clopidogrel, prasugrel, ticagrelor o aspirina > $165 \mathrm{mg}$ diarios), h) sangrado activo o alto riesgo de sangrado que contraindique la anticoagulación, i) hemoglobina $<8 \mathrm{~g} / \mathrm{dl}, \mathrm{j}$ ) plaquetas $<75.000 / \mathrm{mcl}$, o k) clearance de creatinina $<30 \mathrm{ml} / \mathrm{min} / 1,73 \mathrm{~m}^{2}$.

Desde abril 2017 hasta junio 2019 fueron randomizados 1.170 pacientes de 119 centros, 1.155 fueron incluidos en el análisis por intención de tratar modificado, de estos 576 en el grupo apixabán y 579 en el grupo dalteparina. La mediana de edad fue 67 años, $50 \%$ sexo masculino, mediana de peso $75 \mathrm{~kg}$, TEV o embolismo pulmonar sintomático $80 \%$, y el clearance de creatinina $\leq 50 \mathrm{ml} / \mathrm{min} / 1,73 \mathrm{~m}^{2}$ constituyó 8,9\% de la población del grupo apixabán y 10,5\% 
del grupo dalteparina. Los tipos de cáncer en el grupo apixabán fueron: tumor sólido 94,3\% (colorrectal $21 \%$, pulmonar $18,2 \%$, mamario $13,7 \%$, genitourinario $11,5 \%$, ginecológico $10,4 \%$, pancreático o hepatobiliar $7,6 \%$, gastrointestinal superior $4 \%$, cabeza y cuello $2,4 \%$, tejido óseo/blando $1,9 \%$, melanoma de piel $0,7 \%$, otro $2,8 \%$ ), y malignidad hematológica 5,7\%. El Dr. Agnelli destacó durante la presentación que las características de la población eran muy similares a la práctica cotidiana, encontrándose bien representado el cáncer colorrectal.

El resultado primario de eficacia (TEV recurrente) ocurrió en 32 pacientes del grupo apixabán $(5,6 \%)$ y en 46 pacientes de grupo dalteparina (7,9\%) (HR: 0,63; IC: 0,37-1,07; p<0,001 para no inferioridad; $p=0,09$ para superioridad). Ocurrió sangrado mayor en 22 pacientes del grupo apixabán $(3,8 \%)$ y en 23 del grupo dalteparina (4\%) (HR: 0,82; IC: 0,40-1,69; $\mathrm{p}=0,6)$. Episodios de sangrado mayor gastrointestinal ocurrieron en 11 pacientes del grupo apixabán $(1,9 \%)$ y en 10 pacientes del grupo dalteparina $(1,7 \%)$. No existieron episodios de sangrado fatal en el grupo apixabán y existieron dos en el grupo dalteparina. Episodios de sangrado no relevante ocurrieron en 52 pacientes del grupo apixabán (9\%) y en 35 del grupo dalteparina (6\%) (HR: 1,42; IC: 0,88-2,30), siendo el sangrado en el sistema genitourinario y en las vías aéreas superiores, la razón primaria del aumento de la incidencia en el grupo apixabán. La muerte por cualquier causa al día 210 ocurrió en 135 pacientes en el grupo apixabán $(23,4 \%)$ y en 153 en el grupo dalteparina $(26,4 \%)$; en la mayoría asociada al cáncer $(85,2 \%$ en el grupo apixabán y $88,2 \%$ en el grupo dalteparina). En el análisis de subgrupos se encontró que en los pacientes menores de 65 años existía un mayor beneficio de apixabán sobre dalteparina al prevenir TEV recurrente, lo cual es un generador de hipótesis que será estudiado en el futuro.

Las principales limitaciones del estudio son el tener un diseño abierto, no haber preespecificado como resultado el sangrado gastrointestinal, y no haber incluido, por razones de seguridad, pacientes con tumores y metástasis cerebrales o con leucemia aguda. Al concluir su presentación, el Dr. Giancarlo Agnelli resumió: "Apixabán no fue inferior a dalteparina para el tratamiento del TEV asociado al cán- cer, y no se observó un aumento en el riesgo de sangrado mayor, particularmente gastrointestinal. Los hallazgos del estudio Caravaggio amplían la proporción de pacientes con trombosis asociada al cáncer que son elegibles para el tratamiento con anticoagulantes orales directos, incluidos los pacientes con cáncer gastrointestinal".

El editorial que acompañó al artículo en The New England Journal of Medicine, escrito por el Dr. Agnes Lee, destaca que "la evidencia actual presenta un argumento convincente para agregar apixabán como otra opción anticoagulante para el tratamiento del TEV en pacientes con cáncer, sin embargo, dada la heterogeneidad de los ensayos clínicos disponibles, no es apropiado concluir que un anticoagulante oral directo es mejor que otro, sin una comparación cabeza a cabeza" (4). Por lo que destaca el cuidado que debe tenerse al seleccionar el anticoagulante en este tipo de pacientes, apoyándose en el análisis de una historia clínica detallada, tipo, estadio y tratamiento del cáncer, riesgo de sangrado, medicamentos concomitantes, experiencia personal y valores del paciente.

\section{Bibliografía}

1. Levitan N, Dowlati A, Remick SC, Tahsildar HI, Sivinski LD, Beyth R, et al. Rates of initial and recurrent thromboembolic disease among patients with malignancy versus those without malignancy. Risk analysis using Medicare claims data. Medicine (Baltimore). 1999 Sep;78(5):285-91.

2. Farge D, Frere C, Connors JM, Ay C, Khorana AA, Munoz A, et al. 2019 international clinical practice guidelines for the treatment and prophylaxis of venous thromboembolism in patients with cancer. Lancet Oncol. 2019 Oct;20(10):e566-e581. doi: 10.1016/S1470-2045(19)30336-5

3. Agnelli G, Becattini C, Meyer G, Muñoz A, Huisman M, Connors J, et al. Apixaban for the Treatment of Venous Thromboembolism Associated with Cancer. N Engl J Med 2020 Mar 29; [e-pub]. doi: 10.1056/NEJMoa1915103

4. Lee A. Anticoagulant Therapy for Venous Thromboembolism in Cancer. N Engl J Med 2020 Mar 29; [e-pub]. doi: 10.1056/NEJMe2004220 
Ten-year Outcomes After Drug-eluting Stents Versus Coronary Artery Bypass Grafting for Left Main Coronary Disease: Extended Follow Up of the PRECOMBAT Trial

Dado que este estudio remite a decisiones de gran relevancia en el ámbito de los procedimientos cardiológicos invasivos, es útil realizar un breve repaso de sus antecedentes.

Desde antes de la publicación del estudio SYNTAX ${ }^{(1)}$ y su seguimiento a $3^{(2)}$ y 5 años ${ }^{(3)}$, existe debate sobre cuál es el método de revascularización más apropiado en la enfermedad coronaria con lesión no protegida significativa del tronco principal izquierdo (TPI). SYNTAX examinó 1.800 pacientes con lesión del TPI o tres vasos, randomizados 1:1 a intervención percutánea (ICP) con stents liberadores de paclitaxel o cirugía de revascularización miocárdica (CRM), estratificando los resultados según el score SYNTAX, que expresa la complejidad anatómica de la enfermedad. En el grupo global, a 5 años se comprobó una incidencia significativamente menor de principales eventos adversos cardiovasculares y cerebrovasculares mayores (MACCE) en el grupo CRM, sin diferencias en cuanto a ataque cerebrovascular (ACV) y mortalidad, y una definida interacción con el rango de puntaje. En la cohorte con lesión de TPI sólo hubo diferencias significativas a favor de la CRM en el rango más elevado de complejidad lesional.

Entre 2016 y 2019 se publicaron los estudios EXCEL y NOBLE, ambos prospectivos randomizados, integrados por pacientes con lesión de TPI. El EXCEL a $3^{(4)}$ y 5 años ${ }^{(5)}$, fue un estudio de no inferioridad que utilizó stents liberadores de everolimus y reclutó pacientes con score SYNTAX bajo o intermedio, encontrando una incidencia similar de MACCE al término de 5 años, aunque menores tasas de muerte total y de requerimiento de nueva revascularización para el grupo CRM. NOBLE también fue concebido como un estudio de no inferioridad, aunque sin estratificación por score SYNTAX. A 5 años se registró una significativa menor incidencia de MACCE, infarto de miocardio no relacionado al procedimiento y nueva revascularización en el grupo CRM, sin diferencias significativas en muerte y $\mathrm{ACV}^{(6)}$.

Un metaanálisis publicado en 2017 que incluyó 6 estudios y 4.686 pacientes randomizados con estenosis de TPI no encontró diferencias significativas en mortalidad total ni cardiovascular (CV) luego de un seguimiento medio de 39 meses. Sin embargo, estratificando la población por score SYNTAX, la mortalidad CV fue similar con score intermedio, mayor para el grupo CRM con score bajo y mayor para el grupo ICP con score alto. La tasa global de MACCE fue similar, con una previsible interacción con el tiempo, de modo que resultó mayor con CRM dentro de los primeros 30 días del procedimiento y mayor con ICP luego de este período. La revascularización repetida resultó claramente más frecuente con ICP que con $\mathrm{CRM}^{(7)}$.

La comparación del comportamiento de las dos técnicas durante un plazo más prolongado mediante un diseño prospectivo y randomizado se materializó por primera vez con la publicación del estudio SYNTAXES (SYNTAX Extended Survival) en 2019, con seguimiento a 10 años ${ }^{(8)}$. El análisis se centró en la mortalidad por todas las causas como punto final primario, la cual resultó significativamente menor para el grupo CRM con lesiones de 3 vasos, y cuando el análisis se efectuó según la complejidad lesional, para el segmento de mayor score. En cambio, los pacientes con lesión del TPI no exhibieron diferencias en mortalidad con una u otra técnica, aunque a diferencia del SYNTAX a 5 años, no se muestra un análisis según terciles de complejidad anatómica.

El estudio presentado el día de ayer corresponde a un seguimiento a 10 años del estudio PRECOMBAT, publicado en $2015^{(9)}$. Se trata de un estudio prospectivo, randomizado, multicéntrico (13 centros de Corea del Sur), que reclutó entre abril de 2004 y agosto de 2009, 600 pacientes de 1.454 elegibles con lesión del $\mathrm{TPI} \geq 50 \%$ y diferentes manifestaciones clínicas de isquemia, luego de aplicar varios criterios de exclusión.

Los equipos de cardiología intervencionista y cirugía cardíaca estuvieron de acuerdo en que los pacientes eran tratables tanto por ICP como por CRM, y fueron randomizados en una relación 1:1 para uno $\mathrm{u}$ otro procedimiento. Las poblaciones estuvieron equilibradas en cuanto a antecedentes clínicos, manifestaciones de isquemia, EuroSCORE, función ventricular, número de vasos afectados y score SYNTAX (evaluado post hoc).

En ICP se utilizaron stents de sirolimus, y se indicó antiagregación dual durante al menos un año. La CRM se realizó sin circulación extracorpórea en el $64 \%$, y se utilizó la arteria mamaria izquierda en el 93.6\% de los casos. Se implantaron 2,7 1,4 stents y se confeccionaron $2,7 \pm 0,9$ bypass por paciente. La revascularización se consideró completa en el $68,3 \%$ para ICP y $70,3 \%$ para CRM.

El punto final primario fue un objetivo compuesto de mortalidad total, infarto de miocardio (IM), ACV o isquemia de vaso revascularizado. Los puntos secundarios incluyeron: los puntos individuales previamente considerados, un objetivo compuesto de muerte, IM o ACV, necesidad de cual- 
quier revascularización y trombosis del stent y oclusión sintomática de bypass. Los resultados se analizaron según intención de tratamiento.

En cuanto a los resultados, el punto final primario se cumplió en el $29,8 \%$ para ICP y $24,7 \%$ para CRM (HR: 1,25; IC: 0,93-1,69), lo que indica una tendencia favorable para CRM sin significación estadística. El punto combinado de muerte, IM o ACV tampoco se diferenció: 18,2\% para ICP y 17,5\% para CRM (HR: 1,00; IC: 0,70-1,44). Lo mismo ocurrió para la mortalidad total: $14,5 \%$ para ICP y $13,8 \%$ para CRM (HR: 1,13 ; IC $0,75-1,70$ ). En cambio, y como se ha comprobado en casi todos los estudios previos, con ICP se duplicó la tasa de requerimiento de nueva revascularización: $16,1 \%$ vs. $8,0 \%$ (HR: 1,98 ; IC: $1,21-3,21$ )

A primera vista, la ausencia de un beneficio diferencial de la CRM a 10 años más allá de su protección antiisquémica más prolongada podría resultar algo sorprendente. Teniendo en consideración los resultados de los estudios previos, donde las curvas de sucesos mayores tienden a separarse luego de los 5 años, podría esperarse una ventaja de la CRM en eventos "duros". Sin embargo, este hecho puede explicarse, en primer lugar porque se trata de un estudio pequeño, con limitado poder estadístico para detectar diferencias significativas en la incidencia de eventos. Por otra parte, el análisis por intención de tratamiento llevó a un cruzamiento de pacientes de un $25 \%$, con lo cual la posibilidad de atenuar una diferencia real se incrementa. En este sentido, debe destacarse que en la publicación online del estudio en Circulation, los autores analizan los datos según el tratamiento efectivamente efectuado (por protocolo), incluyendo 276 pacientes aleatoriamente asignados a ICP y 248 a CRM que se mantuvieron en sus respectivos grupos. En este análisis hubo un HR del punto final primario para ICP vs. CRM de 1,51, con IC: 1,09-2,10, con lo que se abre un margen para un beneficio más sólido si el estudio fuera de mayor porte.

Por otro lado, dado el momento de reclutamiento de pacientes, no fueron utilizados stents hoy considerados de última generación, que podrían mejorar los resultados de la ICP. Sin embargo, con respecto a este punto cabe recordar un registro también procedente de Corea con seguimiento a 10 años ${ }^{(10)}$, donde si bien no se encontró diferencias en el punto combinado al término del período, en la cohorte que compara ICP con stents fármacoactivos versus CRM realizada en la misma etapa, la ICP se asoció a mayor riesgo de muerte y severos eventos finales compuestos a los 5 años. Curiosamente, los autores no observaron este beneficio en la era de los stents metálicos, lo que atribuyen a la expansión re- ciente de la intervención percutánea a casos de mayor complejidad clínica y anatómica.

En definitiva, el debate con respecto al beneficio de una u otra técnica con respecto a mortalidad continúa abierto, y si bien son bienvenidos los estudios controlados de largo aliento, son aún susceptibles de sesgos metodológicos y de interpretación. Por otra parte, los rápidos cambios en las técnicas, dispositivos y farmacología adyuvante generan una dificultad adicional para obtener conclusiones persistentes, aunque ello constituye un mal menor en comparación con el beneficio en la expectativa y calidad de vida que indudablemente han ofrecido a los pacientes.

\section{Bibliografía}

1. Serruys PW, Morice M-C, Kappetein A P, Colombo A, Holmes DR, Mack MJ, et al, for the SYNTAX Investigators. Percutaneous Coronary Intervention versus Coronary-Artery Bypass Grafting for Severe Coronary Artery Disease. N Engl J Med. 2009; 360: 961-72.

2. Kappetein AP, Feldman TE, Mack MJ, Morice M-C, Holmes DR, Stahle E, et al. Comparison of coronary bypass surgery with drug-eluting stenting for the treatment of left and/or three-vessel disease: 3-year follow-up of the SYNTAX trial. Eur Heart $\mathrm{J}$ 2011; 32: 2125-34.

3. Mohr FW, Morice M-C, Kappetein AP, Feldman TE, Stahle E, Colombo A, et al. Coronary artery bypass graft surgery versus percutaneous coronary intervention in patients with three-vessel disease and left main coronary disease: 5 year follow-up of the randomized, clinical SYNTAX trial. Lancet 2013; 391: 629-38.

4. Stone GW, Sabik JF, Serruys PW, Simonton CA, Généreux P, Puskas J, et al, for the EXCEL Trial Investigators. Everolimus-Eluting stents or Bypass Surgery for Left Main Coronary Artery Disease. N Engl J Med 2016; 375: 2223-35.

5. Stone GW, Kappetein AP, Sabik JF, Pocock SJ, Morice M-C, Puskas J, et al, for the EXCEL Trial Investigators. Five-year outcomes after PCI or CABG for Left Main Coronary Disease. N Engl J Med 2019; 381:1820-30.

6. Mäkikallio T, Holm NR, Lindsay M, Spence MS, Erglis A, Menown IBA, et al, for the NOBLE study investigators. Percutaneous coronary angioplasty versus coronary artery bypass grafting in treatment of unprotected left main stenosis (NOBLE): a prospective, randomised, open-label, non-inferiority trial. Lancet 2019; 388: 2743-52.

7. Palmerini T, Serruys PW, Kappetein AP, Généreux P, Della Riva D, Bacchi Reggiani L, et al. Clinical Outcomes with Percutaneous Coronary Revascularization versus Coronary Artery Bypass Grafting Surgery in Patients with Unprotected Left Main Coronary 
Artery Disease: A Meta-Analysis of Six Randomized Trials and 4686 Patients. Am Heart J 2017; 190: 54-63.

8. Thuijs D, Kappetein AP, Serruys PW, Mohr FW, Morice M-C, Mack MJ, et al. Percutaneous coronary intervention versus coronary artery bypass grafting in patients with three-vessel of left main coronary artery disease: 10-year follow-up of the multicenter randomized controlled SYNTAX trial. Lancet Published Online September 2, 2019. doi.org/10. 1016/S0140-6736(19)31997-X

9. Jung-Min Ahn, Jae-Hyung Roh, Young-Hak Kim, Duk-Woo Park, Sung-Cheol Yun, Pil
Hyung Lee, et al. Randomized Trial of Stents versus Bypass Surgery for Left Main Coronary Artery Disease: Five-Year Outcomes of the PRECOMBAT Study. J Am Coll Cardiol. 2015; 65: 2198-206. doi: 10.1016/j.jacc.2015.03.033

10. Duk-Woo Park, Jung-Min Ahn, Sung-Cheol Yung, Yong-Hoon Yoon, Do-Yoon Kang, Pil Hyung Lee, et al. 10-Year Outcomes of Stents Versus Coronary Artery Bypass Grafting for Left Main Coronary Artery Disease. J Am Coll Cardiol 2018; 72: 2813-22.

\section{Anticoagulation with or without Clopidogrel after Transcatheter Aortic-Valve Implantation: POPular TAVI}

Alrededor de un tercio de los pacientes que reciben un implante de prótesis aórtica transcatéter (TAVI) requiere anticoagulación oral (ACO), fundamentalmente por fibrilación auricular $(\mathrm{FA})^{(1)}$ lo que dificulta la terapia antitrombótica. Las guías clínicas sobre tratamiento antitrombótico sugieren por recomendación de expertos el uso de ACO basada en antagonistas de la vitamina $\mathrm{K}$ a largo plazo, solo o combinado con un antiagregante plaquetario ${ }^{(2,3)}$. Esta combinación pretende reducir el riesgo de complicaciones tromboembólicas asociadas a la prótesis, que pueden ser devastadoras, pero podría incrementar el riesgo de sangrado. Las complicaciones hemorrágicas luego del TAVI ocurren en 3\%$13 \%$ de los pacientes y aumentan la mortalidad, mientras que los ataques cerebrovasculares (ACV) pueden presentarse en 1\%-12\% al año de implantada la prótesis ${ }^{(4,5)}$. Se trata, por lo tanto, de un tema en primera línea de interés para cardiólogos clínicos e intervencionistas.

Los resultados de la cohorte B de POPular TAVI $^{(6)}$ fueron presentados por el Dr. Vincent Nijenhuis y publicados simultáneamente el 29 de marzo en The New England Journal of Medicine. Se trata de un estudio aleatorizado, abierto y multicéntrico, de grupos paralelos, independiente de la industria, realizado en 17 centros europeos de Países Bajos, Bélgica, República Checa y Luxemburgo. POPular TAVI ${ }^{(7)}$ pretende probar que la monoterapia con ácido acetilsalicílico o un anticoagulante oral luego de TAVI es más segura que adicionar clopidogrel durante 3 meses, sin incrementar significativamente las complicaciones tromboembólicas o isquémicas. El estudio presenta un total de 1.000 pacientes seleccionados para TAVI por un heart team, divididos en dos cohortes antes de la aleatorización: la cohorte A cuyos resultados aún no se encuentran disponibles, incluye pacientes sin indicación de
ACO, y la cohorte B que reúne 326 pacientes con indicación previa y permanente de ACO. Se excluyeron pacientes que habían recibido un stent liberador de fármacos en los 3 meses previos, o stent metálico en el mes previo, y aquellos con alergia o intolerancia a clopidogrel. Los pacientes continuaron su ACO habitual. Previo al TAVI, se aleatorizaron 1:1 a recibir o no clopidogrel asociado al ACO durante 3 meses. El procedimiento se realizó según el protocolo de cada centro, recomendándose durante el mismo el uso de heparina no fraccionada. El clopidogrel se administró mediante dosis de carga de $300 \mathrm{mg}$ el día antes del TAVI o el propio día del procedimiento, seguido de $75 \mathrm{mg}$ una vez al día. Los pacientes completaron un cuestionario de seguimiento a los 3,6 y 12 meses respecto a aparición de resultados primarios y secundarios, medicación prescrita, estado de salud y calidad de vida. Los datos de seguimiento fueron evaluados por personas que no conocían la aleatorización, a partir de los cuestionarios y de los registros electrónicos de pacientes.

13 pacientes fueron excluidos luego de aleatorización, incluyéndose finalmente 313 pacientes, de los cuales 156 recibieron ACO y clopidogrel. Fueron seguidos durante al menos un año luego del TAVI. Los puntos finales primarios fueron todos los sangrados, definidos según Valve Academic Research Consortium-2 (VARC-2) y sangrados no relacionados al procedimiento. Como VARC-2 no distingue eventos hemorrágicos relacionados al procedimiento, estos fueron delimitados en base a ítems de la definición de hemorragia severa tipo 4 del Bleeding Academic Research Consortium (BARC). En cuanto a las limitaciones, los autores resaltaron que la mayoría de las hemorragias del sitio de punción clasificadas como VARC-2 se consideraron no relacionadas con el procedimiento. Se definieron dos puntos finales 
secundarios: a) un compuesto de muerte cardiovascular, sangrado no relacionado con el procedimiento, ACV (incluyendo hemorrágico) o infarto de miocardio, $\mathrm{y}$ b) vinculado fundamentalmente a eventos isquémicos, valorado como un compuesto de muerte cardiovascular, ACV (excluyendo hemorragia) o infarto de miocardio.

Los grupos de pacientes tuvieron similares características, con una edad media de $81 \pm 5,9$ años y $45,4 \%$ fueron mujeres. $95 \%$ de los pacientes tenían FA. El 75,2\% utilizaban antagonistas de la vitamina $\mathrm{K}$ y el resto ACO directos. La adherencia al clopidogrel fue $95,5 \%$. No hubo pérdida de pacientes ni datos en el seguimiento. La hemorragia ocurrió en $21,7 \%$ de pacientes que recibieron $\mathrm{ACO}$ versus $34,6 \%$ de los que recibieron doble terapia (RR: 0,63; IC: 0,43-0,90; p=0,011). La mayoría de los eventos hemorrágicos ocurrieron en el sitio de acceso vascular. La hemorragia no relacionada con el procedimiento ocurrió en el $21,7 \%$ en los pacientes con ACO y $34 \%$ en el pacientes con ACO más clopidogrel (RR: 0,64; IC: 0,44-0,92; $\mathrm{p}=0,02$ ). Los sangrados fueron menores y en su mayoría durante el primer mes. Respecto al primer punto final secundario, ocurrió en el 31,2\% del grupo ACO y 45,5\% en el grupo con doble terapia, con una diferencia significativa en el análisis de no inferioridad y superioridad a favor de ACO aislada. El segundo punto final secundario ocurrió en el $13,4 \%$ en el grupo ACO y $17,3 \%$ en los que recibieron ACO más clopidogrel, demostrando no inferioridad, pero sin obtener superioridad significativa. Como conclusión, los pacientes que recibieron TAVI y que tienen indicación de ACO presentan menor incidencia de hemorragia grave con ACO aislada cuando se compara con ACO más clopidogrel. "Los resultados sugieren que sería beneficioso no administrar clopidogrel. Es más seguro porque no conduce a tantos eventos de sangrado", dijo Nijenhuis durante su conferencia.

Yamel Ache, https://orcid.org/0000-0001-9956-4081 Carlos Guamán, https://orcid.org/0000-0002-1065-1988 María Victoria Ramos, https://orcid.org/0000-00026349-2781

Víctor Dayan, https://orcid.org/0000-0002-5470-0585 Jorge Estigarribia, https:/orcid.org/0000-0002-51367668

Este artículo fue aceptado para su publicación por: Editor jefe Dr. Gerardo Soca.

\section{Bibliografía}

1. Brinkert M, Keller LS, Moriyama N, et al. Safety and Efficacy of Transcatheter Aortic Valve Replacement With Continuation of Oral Anticoagulation. J Am Coll Cardiol. 2019 Apr, 73 (15) 2004-5. doi: 10.1016/j.jacc.2018.11.066

2. Baumgartner H, Falk V, Bax JJ, et al. 2017 ESC/EACTS guidelines for the management of valvular heart disease. Eur Heart J 2017;38:2739-91. doi: 10.1093/eurheartj/ehx391

3. Nishimura RA, Otto CM, Bonow RO, et al. 2017 AHA/ACC focused update of the 2014 AHA/ACC guideline for the management of patients with valvular heart disease: a report of the American College of Cardiology/American Heart Association Task Force on Clinical Practice Guidelines. Circulation 2017;135(25):e1159-e1195. doi: 10.1161/CIR.00000 00000000503

4. Nijenhuis VJ, Bennaghmouch $\mathbf{N}$, van Kuijk J-P, et al. Antithrombotic treatment in patients undergoing transcatheter aortic valve implantation (TAVI). Thromb Haemost 2015;5:113(4). doi: 10. 1160/TH14-10-0821

5. Borz B, Durand E, Godin M, et al. Incidence, predictors and impact of bleeding after transcatheter aortic valve implantation using the balloon-expandable Edwards prosthesis. Heart 2013;99(12):860-5. doi: 10.1136/heartjnl-2012-303095

6. Nijenhuis VJ, Bennaghmouch N, Hassell M, et al. Rationale and design of POPular-TAVI: antiPlatelet therapy fOr Patients Undergoing Transcatheter Aortic Valve Implantation. Am Heart J 2016;173: 77-85. doi:10.1016/j.ahj.2015.11.008

7. Nijenhuis VJ, Brouwer J, Delewi R, et al. Anticoagulation with or without Clopidogrel after Transcatheter Aortic-Valve Implantation. NEJM [publicado en línea]. Marzo 29, 2020. Disponible en: https:// www.nejm.org/doi/full/10.1056/NEJMoa1915152?que ry=featured_home. doi: 10.1056/NEJMoa 1915152 